\title{
Atuação do farmacêutico na dispensação de medicamentos em Centros de Atenção Psicossocial Adulto no município de São Paulo, SP, Brasil
}

\author{
The role of the pharmacist in dispensing medication \\ in Adult Psychosocial Care Centers in the city of São Paulo, \\ Capital of the State of São Paulo, Brazil
}

${ }^{1}$ Faculdade de Ciências Farmacêuticas, Universidade de São Paulo. Av. Prof. Lineu Prestes 580/Conj. das Químicas/Bl. 13/Cidade Universitária, Butantã 05508-900 São Paulo SP Brasil. aguiar.pm@usp.br

\begin{abstract}
The objective of this study was to evaluate the role of the pharmacist in dispensing medication by conducting cross-sectional exploratory-descriptive research in eight Adult Psychosocial Care Centers (CAPS) in São Paulo. The pharmacists responsible for each of the dispensing units studied filled out a semi-structured questionnaire about the service provided. Two Adult CAPS units were selected from each of the North, South, East and West regions of São Paulo. The central region has no Adult CAPS, and was therefore not included in the study. Most of the respondents were aged between 35 and 40 years and were predominantly female. It was found that half of the respondents performed only $25 \%$ of dispensations and few conducted an analysis of all prescriptions before dispensing medication. All respondents contacted the prescriber if any medication-related problems arose. However, few pharmaceutical interventions were commonly performed. Furthermore, one respondent indicated that all his/her functions in the pharmacy could be delegated to another professional. These findings reveal the pressing need for actions that ensure the ongoing training of pharmacists to enable them to be clinically prepared to deal with patients with mental disorders. Key words Mental health, Pharmaceutical services, Use of medication
\end{abstract}

Resumo O presente estudo teve por objetivo avaliar a atuação do farmacêutico na dispensação de medicamentos, sendo realizada pesquisa transversal exploratório-descritiva em oito Centros de Atenção Psicossocial (CAPS) Adulto do Município de São Paulo. Questionário semiaberto sobre o serviço de dispensação foi aplicado aos farmacêuticos responsáveis de cada unidade estudada. Foram selecionadas duas unidades CAPS Adulto das regiões Norte, Sul, Leste e Oeste de São Paulo. A região central não dispõe de CAPS adulto, razão pela qual não fez parte do estudo. A maior parte dos entrevistados apresentou idade entre 35 e 40 anos, com predomínio do gênero feminino. Verificou-se que metade dos entrevistados realizou apenas $25 \%$ das dispensações e poucos fizeram a análise de todas as prescrições antes de dispensar o medicamento. Todos os respondentes entravam em contato com o prescritor na presença de algum problema relacionado ao medicamento. Entretanto, intervenções farmacêuticas não eram realizadas comumente. Ainda, um dos respondentes indicou que todas as suas funções na farmácia poderiam ser delegadas a outro profissional. Os dados reforçam a necessidade de ações que possibilitem o aprimoramento contínuo do farmacêutico a fim de que esteja apto à prática clínica voltada aos pacientes com transtornos mentais.

Palavras-chave Saúde mental, Serviços farmacêuticos, Uso de medicamentos 


\section{Introdução}

A reforma psiquiátrica brasileira propõe a substituição do modelo manicomial pela criação de uma rede de serviços substitutivos territorializados, com abordagem direcionada para a atenção psicossocial da comunidade. $\mathrm{Na}$ expansão deste movimento, os Centros de Atenção Psicossocial (CAPS) assumem relevância especial no cenário das novas práticas em saúde mental no País, configurando-se como dispositivo estratégico para a reversão do modelo hospitalar ${ }^{1,2}$.

Os CAPS destinam-se a dar suporte ao paciente com sofrimento mental, envolvendo não só o tratamento clínico, mas uma compreensão da situação que o circunda, com intervenções cujo objetivo é assegurar sua reinserção no contexto social e familiar. Assim, propõe-se um cuidado que tem como premissas a integralidade da atenção e a humanização da assistência ${ }^{3}$. Nesse sentido, torna-se necessária a criação de arranjos que facilitem a aproximação entre profissionais e pacientes, de modo que possibilitem a construção e o acompanhamento de projetos terapêuticos sensíveis às necessidades peculiares do indivíduo ${ }^{4}$.

Um dos arranjos destinados a tal finalidade é o trabalho de referência no CAPS, organizado por meio de profissionais ou equipes de referência, cujo funcionamento se fundamenta na construção conjunta e colaborativa do projeto terapêutico do paciente ${ }^{5}$. Além dos membros que constituem a equipe terapêutica tradicional, pode-se contar com a presença de outros profissionais, dentre eles o farmacêutico ${ }^{1,6}$. Todavia, a atuação deste farmacêutico tem sido limitada às unidades CAPS que dispõem de farmácias que centralizam a distribuição de medicamentos em saúde mental ${ }^{7}$.

Os medicamentos constituem ferramentas poderosas para mitigar o sofrimento humano. Produzem curas, prolongam a vida e retardam o surgimento de complicações associadas às doenças, facilitando o convívio entre o indivíduo e sua enfermidade ${ }^{8}$. Entretanto, fatores relacionados ao processo de utilização dos medicamentos se refletem no efeito terapêutico desejado, e, por isso, nem sempre exercem plenamente sua função. Desta forma, torna-se importante instruir o paciente acerca do uso dos medicamentos psicotrópicos, identificando potenciais barreiras que podem comprometer o sucesso do tratamento".

Atualmente, a profissão farmacêutica está se movendo de um paradigma técnico para outro social, com prestação de serviços focados no usuário de medicamentos ${ }^{10}$. No Brasil, essa transformação paradigmática tem contribuído de forma significativa para a evolução da profissão e criado expectativas nos profissionais em relação à dignificação dessa atividade, oferecendo uma oportunidade de resgate da relação farmacêuticopaciente, há muito tempo perdida nas farmácias ${ }^{11}$.

Dentre as práticas assistenciais desenvolvidas pode-se destacar o serviço de Dispensação Farmacêutica. Esta, não configura apenas a ocasião em que determinada receita ou prescrição é aviada. Não é troca de mercadorias por receita médica. Segundo Pepe e Castro ${ }^{8}$, tão ou mais importante que o medicamento recebido pelo paciente, é a informação envolvida neste processo. O fluxo da informação, previamente iniciado na consulta médica, tem continuidade no recinto da farmácia.

Durante o ato da dispensação, o farmacêutico deve informar e orientar o paciente sobre o uso adequado dos medicamentos, com ênfase no cumprimento da farmacoterapia, interação com outros medicamentos, alimentos e exames laboratoriais, reconhecimento de reações adversas potenciais e condições de conservação do produto $^{12}$. Esta função informativa e educativa da dispensação torna-a peça chave na cadeia da assistência à saúde e constitui uma das últimas oportunidades de, ainda dentro do sistema de saúde, identificar, corrigir ou reduzir possíveis riscos associados à farmacoterapia ${ }^{13}$.

Estudos sobre a dispensação de medicamentos ainda são escassos no Brasil, sobretudo no âmbito da saúde mental. Pouco se conhece sobre a inserção do profissional farmacêutico no contexto do CAPS e suas ações e intervenções visando ao uso racional de psicotrópicos. Assim, o presente trabalho teve como objetivo avaliar a atuação do farmacêutico na dispensação de medicamentos para pacientes com transtornos mentais em CAPS Adulto do Município de São Paulo.

\section{Material e métodos}

O trabalho corresponde a estudo transversal exploratório-descritivo, realizado entre janeiro e março de 2012, sobre a dispensação nos CAPS Adulto do Município de São Paulo.

\section{Seleção dos CAPS Adulto}

Foi realizado levantamento sobre o número de CAPS existentes no Município de São Paulo 
e verificou-se que, em julho de 2011, havia 27 unidades vinculadas à Secretaria Municipal de Saúde.

Estabeleceu-se o critério de selecionar ao acaso oito das 27 unidades $(29,6 \%)$ para compor a amostra da pesquisa, sendo duas CAPS Adulto de cada uma das regiões: Norte, Sul, Leste e Oeste do Município de São Paulo. A região central não dispõe de CAPS Adulto e, portanto, nenhuma unidade nessa região foi selecionada para o estudo.

Por tratar-se de estudo preliminar e pelo fato de não existir outro semelhante publicado na literatura, considerou-se que oito CAPS representariam uma amostra adequada para abordar o tema, viabilizando o desenvolvimento da pesquisa de campo. Foram incluídos no estudo os CAPS Adulto que funcionavam como uma central de distribuição de medicamentos controlados e dispunham do profissional farmacêutico, dado que foi confirmado mediante ligações telefônicas realizadas pelo pesquisador para a recepção de cada unidade.

Levando-se em consideração os critérios anteriormente citados, as unidades selecionadas para o presente estudo foram: A e B (Zona Oeste), C e D (Zona Leste), E e F (Zona Sul) e G e H (Zona Norte), sendo que os locais C e F são CAPS Adulto III e os demais, CAPS Adulto II.

\section{Coleta e Análise dos dados}

As informações do estudo foram obtidas por meio da aplicação de questionário semiaberto aos farmacêuticos responsáveis de cada uma das oito unidades CAPS Adulto selecionadas. O instrumento foi organizado em blocos temáticos relacionados aos dados socioeconômicos e demográficos e à atuação desse profissional.

Todas as variáveis socioeconômicas e demográficas foram coletadas por meio de questões fechadas, sendo: gênero (dicotômica) e grau de escolaridade, faixa etária e renda ativa total (categóricas). As informações sobre a atuação do profissional farmacêutico foram coletadas empregando-se questões fechadas e abertas.

- Para as questões fechadas foram consideradas as seguintes variáveis: a) Cargo na unidade; realização de plantões; contato com o médico quando detectado algum problema relacionado com medicamentos; e, fornecimento de orientação específica quanto ao modo de utilização e precauções do uso dos medicamentos (dicotômicas); b) Carga horária diária (contínua); c) Tempo de trabalho na unidade; frequência com que a dispensação dos medicamentos é realizada pelo próprio farmacêutico; frequência de análise das prescrições antes da dispensação; e, frequência com que as intervenções farmacêuticas são realizadas (categóricas).

- Algumas questões abertas foram aplicadas a fim de investigar as medidas adotadas pelo profissional, no sentido de contribuir com a adesão do paciente ao tratamento, bem como avaliar a opinião do farmacêutico sobre as funções que não poderiam ser delegadas a outros profissionais.

Os dados coletados foram reunidos em banco de dados, realizando-se análise descritiva por meio do programa estatístico Epi Info versão 3.5.2. O presente estudo foi aprovado pelos Comitês de Ética em Pesquisa da Faculdade de Ciências Farmacêuticas da Universidade de São Paulo e da Secretaria Municipal de Saúde de São Paulo.

\section{Resultados}

Apesar da confirmação efetuada por meio de ligação telefônica, de que a dispensação dos medicamentos controlados é realizada por farmacêutico, observou-se que em uma unidade CAPS Adulto da Zona Sul não havia a presença deste profissional. Esta ocorrência pode ser explicada pelo fato de que esse CAPS é administrado por um hospital da região, sendo que a farmacêutica do hospital não tem contato com os pacientes atendidos na unidade. Apenas é feito o envio de medicamentos de emergência para a unidade, os quais são entregues aos pacientes por uma profissional de enfermagem. Por esta razão, a referida farmacêutica não respondeu a maior parte das questões propostas no questionário.

A maioria dos farmacêuticos apresentou idade entre 35 e 40 anos e houve predomínio do gênero feminino $(87,5 \%)$. Mais da metade cursou uma pós-graduação e apresentou renda ativa igual ou acima de 5 salários mínimos. Grande parte dos profissionais trabalhava na unidade há 1-3 anos, não realizava plantões e exercia o cargo de farmacêutico. Todos os respondentes do questionário trabalhavam 8 horas diárias (Tabela1).

Quanto à prática profissional, verificou-se que metade dos respondentes realizava apenas $25 \%$ das dispensações. Somente três farmacêuticas analisavam todas as prescrições, porém uma delas o fazia após dispensar o medicamento. Todos os profissionais farmacêuticos entravam em contato com o prescritor na presença de algum problema relacionado ao medicamento. Intervenções farmacêuticas não eram realizadas habitualmente e, quando ocorriam, estavam rela- 
cionadas, sobretudo, à qualidade e à quantidade da farmacoterapia prescrita. As orientações eram feitas de forma verbal (contato direto ou ligação telefônica) ou escrita para o psiquiatra ou equipe multiprofissional (Tabela 2).

Quando os farmacêuticos foram questionados sobre as medidas adotadas a fim de contribuir com a adesão do paciente ao tratamento, a maioria buscava esclarecer sobre a doença ou a indicação e benefícios do tratamento medicamentoso. Exclusivamente, uma farmacêutica utilizava ferramentas lúdicas e ilustrativas para facilitar o uso correto do medicamento (Quadro 1).

No geral, os farmacêuticos indicaram que diversas funções na farmácia não poderiam ser delegadas a outros profissionais, as quais variam desde serviços técnico-gerenciais, como o controle do estoque, até atividades desenvolvidas junto ao paciente visando ao uso racional de me- dicamentos. Em contrapartida, um respondente afirmou que todas as suas atividades poderiam ser delegadas a outros profissionais (Quadro 2).

Além das questões relativas ao perfil e à atuação profissional, facultou-se aos entrevistados acrescentar informações que julgassem pertinentes. Duas das farmacêuticas o fizeram conforme destacado a seguir:

Os farmacêuticos do CAPS devem receber maiores e melhores orientações do setor de Assistência Farmacêutica Municipal, visando à melhoria na qualidade do atendimento e fortalecendo sua atuação farmacêutica nas unidades. Procedimentos homogêneos também são necessários para padronizar a atuação em todas as unidades. Gerências de profissionais não farmacêuticos dificulta a atuação (Farmacêutica CAPS Adulto D).

O farmacêutico deve demonstrar que faz parte da equipe multiprofissional, discutir os casos,

Tabela 1. Caracterização dos farmacêuticos quanto a variáveis pessoais e profissionais em oito CAPS Adulto do Município de São Paulo, Brasil, 2012.

\begin{tabular}{|c|c|c|c|c|c|c|c|c|}
\hline \multirow[b]{2}{*}{ Variável } & \multicolumn{2}{|c|}{ Zona Oeste } & \multicolumn{2}{|c|}{ Zona Leste } & \multicolumn{2}{|c|}{ Zona Sul } & \multicolumn{2}{|c|}{ Zona Norte } \\
\hline & $\mathbf{A}$ & B & $\mathrm{C}$ & $\mathrm{D}$ & $\mathbf{E}$ & F & G & $\mathbf{H}$ \\
\hline Faixa etária (anos) & $\geq 45$ & $\geq 45$ & $<30$ & $40-44$ & $35-39$ & $30-34$ & $35-39$ & $35-39$ \\
\hline Gênero (F ou M) & $\mathrm{M}$ & $\mathrm{F}$ & $\mathrm{F}$ & $\mathrm{F}$ & $\mathrm{F}$ & $\mathrm{F}$ & $\mathrm{F}$ & $\mathrm{F}$ \\
\hline Grau de escolaridade & G & PG & G & G & PG & PG & PG & PG \\
\hline Tempo na unidade (anos) & $\geq 7$ & $\geq 1-3$ & $\geq 1-3$ & $\geq 1-3$ & $\ldots$ & $<1$ & $\geq 1-3$ & $\geq 7$ \\
\hline Carga diária (horas) & 8 & 8 & 8 & 8 & $\ldots$ & 8 & 8 & 8 \\
\hline Realiza plantões & Não & Não & Não & Não & Não & Sim & Não & Não \\
\hline Renda ativa total (salários mínimos) & $\geq 5$ & $\geq 5$ & 3 a 4 & 3 a 4 & $\geq 5$ & $\ldots$ & $\geq 5$ & $\geq 5$ \\
\hline Cargo na unidade & FR & FR & FR & FR & FR & FR & FR & Ge FR \\
\hline
\end{tabular}

Notas: F - Feminino; M - Masculino; G - Graduação; PG - Pós-Graduação; (...) - Não se dispõe do dado; FR - farmacêutico responsável e $\mathrm{G}$ - gerente.

Tabela 2. Respostas dos farmacêuticos responsáveis quanto a sua prática profissional em oito CAPS Adulto do Município de São Paulo, Brasil, 2012.

\begin{tabular}{|c|c|c|c|c|c|c|c|c|}
\hline \multirow[b]{2}{*}{ Pergunta } & \multicolumn{2}{|c|}{ Zona Oeste } & \multicolumn{2}{|c|}{ Zona Leste } & \multicolumn{2}{|c|}{ Zona Sul } & \multicolumn{2}{|c|}{ Zona Norte } \\
\hline & A & B & C & D & E & F & G & $\mathbf{H}$ \\
\hline 1 & $25 \%$ & $25 \%$ & $50 \%$ & $50 \%$ & $\ldots$ & $25 \%$ & $100 \%$ & $25 \%$ \\
\hline 2 & $50 \%$ & $100 \%^{*}$ & $50 \%$ & $100 \%$ & $\ldots$ & $50 \%$ & $100 \%$ & $50 \%$ \\
\hline 3 & Às vezes & Sim, sempre & Às vezes & Não & $\ldots$ & Às vezes & Sim, sempre & Sim, sempre \\
\hline 4 & Sim & Sim & Sim & Sim & $\ldots$ & Sim & Sim & Sim \\
\hline 5 & Sim & Sim & Sim & Sim & $\ldots$ & Sim & Sim & Sim \\
\hline
\end{tabular}

Notas:

1 - Realiza todas as dispensações?

2 - Analisa as prescrições antes de dispensar?

3 - Realiza intervenção farmacêutica?

4 - Entra em contato com médico quando detecta problema relacionado ao medicamento?

5- Durante a dispensação fornece orientação específica quanto ao modo de utilização e precauções do uso da medicação?

* A farmacêutica analisa as prescrições após a dispensação.

(...) - Não se dispõe do dado. 
Quadro 1. Respostas dos farmacêuticos responsáveis à pergunta "Quais medidas são adotadas por você que objetivam contribuir com a adesão do paciente ao tratamento?", em oito CAPS Adulto do Município de São Paulo, Brasil, 2012.

\begin{tabular}{|l|l|}
\hline Zona Oeste & $\begin{array}{l}\text { Orientações Gerais (CAPS Adulto A). } \\
\text { Orientação detalhada e sistemática na hora da dispensação (CAPS Adulto B). }\end{array}$ \\
\hline Zona Leste & $\begin{array}{l}\text { [Através de] orientações sobre a indicação e benefícios do uso do medicamento. Uso de } \\
\text { ferramentas lúdicas e ilustrativas que facilite o uso correto do medicamento. Para aqueles } \\
\text { pacientes que apresentam dificuldade de autoadministração e que não possuem a atenção de } \\
\text { um familiar ocorre o fracionamento e separação do medicamento em caixas contendo o nome } \\
\text { do paciente, o que obriga a comparecer ao CAPS para ser monitorado e para administrar a } \\
\text { medicação (CAPS Adulto C). } \\
\text { Orientação sobre a patologia e a importância do tratamento medicamentoso (CAPS Adulto D). }\end{array}$ \\
\hline Zona Sul & $\begin{array}{l}\text { Orientação sobre a necessidade de melhora no quadro da patologia, benefícios em relação à } \\
\text { adesão medicamentosa (CAPS Adulto F). }\end{array}$ \\
\hline Zona Norte & $\begin{array}{l}\text { Atendimento periódico com pacientes e familiares orientados quanto a importância da terapia } \\
\text { medicamentosa (CAPS Adulto G). } \\
\text { Aconselhamento nos grupos de família e [acompanhamento] da assiduidade de paciente em } \\
\text { retirar o medicamento na farmácia (CAPS Adulto H). }\end{array}$ \\
\hline
\end{tabular}

Quadro 2. Respostas dos farmacêuticos responsáveis à pergunta "Em sua opinião, qual a função do farmacêutico em uma farmácia, que não poderia ser delegada a outro profissional?”, em oito CAPS Adulto do Município de São Paulo, Brasil, 2012.

\begin{tabular}{|l|l|}
\hline Zona Oeste & $\begin{array}{l}\text { As exclusivas do farmacêutico conforme edital do concurso público de SMS e as designadas } \\
\text { pelo conselho de ética profissional (CAPS Adulto B). } \\
\text { Nenhuma (CAPS Adulto A). }\end{array}$ \\
\hline Zona Leste & $\begin{array}{l}\text { Proporcionar atenção farmacêutica adequada e demonstrar o uso racional de medicamentos } \\
\text { (CAPS Adulto D). }\end{array}$ \\
\hline Zona Sul & $\begin{array}{l}\text { Atenção farmacêutica, controle e armazenamento do estoque, responsabilidade técnica } \\
\text { conforme RDC 344/98 (CAPS Adulto F). }\end{array}$ \\
\hline Zona Norte & $\begin{array}{l}\text { Dispensação (CAPS Adulto G). } \\
\text { Manipulação de medicamentos, controle de qualidade, [orientar sobre] uso racional de } \\
\text { medicamentos e fiscalização dos outros profissionais (CAPS Adulto H). }\end{array}$ \\
\hline
\end{tabular}

mostrar para a equipe que ele é um profissional em destaque que pode e faz a diferença e não é apenas um dispensador de remédio (Farmacêutica CAPS Adulto $\mathrm{H})$.

\section{Discussão}

De acordo com a Portaria SVS/MS no 344, de 12 de maio de $1998^{14}$, o farmacêutico é o profissio- nal responsável pela dispensação de medicamentos controlados, que deve ocorrer com a sua autorização, após a avaliação da prescrição e da notificação de receita. Apesar disso, verificou-se que nas unidades CAPS estudadas a dispensação não era rotineiramente realizada por este profissional e apenas dois dos farmacêuticos entrevistados realizavam a análise de todas as prescrições médicas antes de dispensar o medicamento. Logo, uma das atividades mais importantes do ciclo da 
Assistência Farmacêutica não está ocorrendo de forma integral, o que pode comprometer a qualidade do cuidado prestado ao usuário de medicamentos.

Na unidade CAPS em que não há atuação direta do farmacêutico, a ação que corresponde à dispensação era realizada por um enfermeiro. Ainda que o estabelecimento distribuísse alguns medicamentos com prescrição médica exclusivamente em situações de emergência, tal procedimento não possuía amparo legal. Mesmo que se classifique a "farmácia básica" como dispensário (estoque), isto não a desobriga de contratar um farmacêutico devido ao fato de haver distribuição de medicamentos controlados ${ }^{14}$.

Antes de proceder a dispensação de psicofármacos, o farmacêutico deve averiguar questões referentes à legibilidade e à legalidade, de acordo com as disposições da Portaria no 344/98. A observação da identificação do emitente e do usuário, a presença da assinatura e carimbo, o nome do medicamento de acordo com a Denominação Comum Brasileira, a quantidade, a forma farmacêutica, a dose e a posologia do medicamento são essenciais para garantir a segurança da dispensação. Tais informações são fundamentais para que o farmacêutico possa orientar o paciente sobre a forma de uso correto do medicamento ${ }^{15}$.

Embora todos os entrevistados informassem contatar o médico quando algum problema com a farmacoterapia era detectado, nem todos realizavam intervenções nesse sentido. Este fato pode indicar que o farmacêutico ainda não se sente inteiramente à vontade para discutir com o médico a farmacoterapia prescrita, o que pode comprometer a colaboração entre os profissionais. Problemas nessa relação afetam negativamente os resultados em saúde do paciente e podem levar a falhas no uso dos medicamentos ${ }^{16}$. Por isso, estudos têm indicado que o desenvolvimento e a manutenção de relações cooperativas entre farmacêuticos e médicos facilitariam a mudança da prática em farmácias ${ }^{17}$. Segundo Montgomery et al. ${ }^{18}$, o sucesso desta cooperação depende de que ambas as partes estejam cientes e concordem com as respectivas responsabilidades profissionais.

Os resultados deste estudo, entretanto, indicam que nem todos os farmacêuticos estão cientes e seguros quanto à sua atribuição e importância junto à equipe de saúde. Como pode ser verificado no Quadro 2, um dos respondentes apontou que todas as suas atividades na farmácia poderiam ser delegadas a outros profissionais. Em contrapartida, como informado anteriormente, um farmacêutico indicou que sua atuação não deve se limitar à dispensação, o que evidencia um movimento que busca o reconhecimento deste profissional em equipes multidisciplinares de saúde, assim como na comunidade ${ }^{19}$.

Para que haja efetiva mudança da prática profissional em direção ao cuidado da população, a formação e a capacitação dos profissionais farmacêuticos são essenciais ${ }^{17}$. No Brasil, disciplinas relacionadas à Assistência Farmacêutica, com ênfase em práticas clínicas, só foram implantadas a partir de ano de 2002, com a reforma curricular que instituiu as novas diretrizes para os Cursos de Farmácia, ressaltando a necessidade de formação generalista e humanista ${ }^{20}$. Possivelmente, nem todos os entrevistados graduaram-se após a implantação do currículo generalista ou cursaram uma pós-graduação nesta área.

Neste contexto, a educação continuada dos profissionais também é fundamental, sobretudo quando se considera a grade curricular excessivamente tecnicista e incipiente na área clínica, a que foram expostos os graduados antes da mudança curricular. Esta necessidade de aprimoramento foi destacada por uma das respondentes, a qual afirmou que melhores orientações sobre a Assistência Farmacêutica seriam necessárias para promover a melhoria da qualidade de atendimento nas unidades. Contudo, levando-se em consideração que para a formação do farmacêutico com perfil requerido para esta prática é essencial a mudança de comportamentos e de entendimentos sobre as abordagens clínica e holística dos pacientes, tempo e esforços ainda são necessários ${ }^{7}$.

A atuação do farmacêutico no âmbito da saúde mental também requer conhecimentos e habilidades específicas. Indivíduos com transtornos mentais e em uso de psicotrópicos têm, comumente, dificuldade em seguir o regime terapêutico proposto e apresentam alto risco de desenvolver problemas relacionados a medicamentos ${ }^{21}$. Diversas barreiras podem afetar a adesão, como tratamento de longo prazo, efeitos colaterais dos medicamentos e conflito entre a proposta terapêutica e a preferência do próprio usuário ${ }^{22}$.

Algumas dessas barreiras à adesão estão relacionadas diretamente ao paciente e, assim, a orientação transmitida por meio da educação e da conscientização constitui importante passo para a melhoria dos resultados em saúde ${ }^{9,23}$. Neste estudo, os entrevistados foram pouco específicos sobre as estratégias utilizadas e as informações oferecidas aos pacientes durante a dispensação. Este dado pode refletir o fato de os farmacêuticos sentirem menos confiança e menor propensão em fornecer 
informações para pessoas com problemas de saúde mental, em relação àqueles com doenças cardiovasculares, diabetes ou asma ${ }^{24}$. Por outro lado, uma das entrevistadas informou que utilizava ferramentas lúdicas e ilustrativas para facilitar o uso correto dos medicamentos, estratégias já descritas na literatura para potencializar a adesão ao tratamento entre a população estudada ${ }^{25}$.

Os farmacêuticos se encontram em posição ideal para reconhecer os efeitos colaterais, oferecer educação, motivação e propor acompanhamento farmacoterapêutico para melhorar a adesão ao tratamento e a qualidade de vida do paciente ${ }^{23}$. Porém, a incorporação de ações de monitoramento e de avaliação do tratamento farmacológico dos diversos transtornos mentais é uma necessidade ainda não atendida nos serviços que constituem a assistência em saúde mental. As ações em saúde estão voltadas, especialmente, para a disponibilidade de medicamentos, sem necessariamente estarem associadas à racionalização de seu uso ${ }^{26}$.

Discutir a reorientação da Assistência Farmacêutica como estratégia para aprimoramento de ações no campo da saúde mental é um caminho a ser percorrido para superar este obstáculo. Nesse sentido, debates têm contribuído para articular a Política de Assistência Farmacêutica e de Saúde Mental visando qualificar a oferta do acesso integral a medicamentos efetivos, seguros e de qualidade. Recentemente, durante a IV Conferência Nacional de Saúde Mental, houve a proposição da inserção e da capacitação de farmacêuticos para atuação exclusiva na Assistência Farmacêutica em saúde mental, com a ampliação da informação crítica e racional sobre o consumo de medicamentos ${ }^{27}$.

Por fim, vale ressaltar que somente a perspectiva do próprio farmacêutico sobre sua atuação foi analisada neste estudo, o que pode levar à obtenção de vieses em algumas respostas, devido à falta de evidências objetivas. Além disso, a avaliação do paciente e/ou familiar em relação à atuação deste profissional seria um aspecto importante a ser avaliado em estudos futuros. A estratégia amostral utilizada no presente estudo pode limitar a generalização dos resultados. Apesar disso, a presente pesquisa trouxe avanços para o conhecimento do tema, tendo em vista que possibilitou a identificação preliminar do perfil dos farmacêuticos que atuam em saúde mental no Município de São Paulo, evidenciando aspectos da prática da dispensação de medicamentos que podem ser aprimorados.

\section{Conclusão}

Os resultados deste estudo indicam que a prática atual de serviços orientados ao paciente está em desenvolvimento em unidades CAPS que contam com a presença do farmacêutico. Na amostra estudada, observou-se que poucos profissionais realizam todas as dispensações e avaliam as prescrições previamente, assim como ainda não é habitual que o farmacêutico discuta com o médico sobre a farmacoterapia, realizando intervenções para potencializar a adesão do paciente ao tratamento.

A maior parte das atribuições destes profissionais ainda está vinculada a práticas gerenciais, o que dificulta a sua atuação junto ao paciente durante a dispensação e afeta a promoção do uso racional de medicamentos. Logo, ressalta-se a necessidade de ações que possibilitem o aprimoramento contínuo do farmacêutico, possibilitando a compreensão do seu papel junto à equipe multidisciplinar para que este profissional esteja apto à prática clínica voltada aos pacientes com transtornos mentais.

\section{Colaboradores}

CG Zanella foi responsável pela coleta, análise, discussão dos dados e redação do artigo. PM Aguiar colaborou na discussão dos dados, na redação do artigo e na revisão crítica do texto. S Storpirtis orientou a realização de todas as etapas do estudo e colaborou na redação do artigo. 


\section{Referências}

1. Brasil. Ministério da Saúde (MS). Departamento de Ações Programáticas Estratégicas, Secretaria de Atenção à Saúde, Ministério da Saúde. Saúde mental no SUS: os centros de atenção psicossocial. Brasília: MS; 2004.

2. Onocko-Campos RT, Furtado JP. Entre a saúde coletiva e a saúde mental: um instrumental metodológico para avaliação da rede de Centros de Atenção Psicossocial (CAPS) do Sistema Único de Saúde. Cad Saude Publica 2006; 22(5):1053-1062.

3. Pinto DM, Jorge MSB, Pinto AGA, Vasconcelos MGF, Cavalcante CM, Flores AZT, Andrade AS. Projeto terapêutico singular na produção do cuidado integral: uma construção coletiva. Texto Contexto Enferm 2011; 20(3): 493-502.

4. Miranda L, Onocko-Campos RT. Análise das equipes de referência em saúde mental: uma perspectiva de gestão da clínica. Cad Saude Publica 2010; 26(6):11531162 .

5. Delfini PSS, Sato MT, Antoneli PP, Guimarães POS. Parceria entre CAPS e PSF: o desafio da construção de um novo saber. Cien Saude Colet 2009; 14(Supl.1):14831492.

6. Nascimento AF, Galvanese ATC. Avaliação da estrutura dos centros de atenção psicossocial do município de São Paulo, SP. Rev Saude Publica 2009; 43(Supl. 1):8-15.

7. Lucchetta RC, Mastroianni PC. Intervenções farmacêuticas na atenção à saúde mental: uma revisão. Rev Ciênc Farm Básica Apl. 2012; 33(2):165-169.

8. Pepe VLE, Castro CGSO. A interação entre prescritores, dispensadores e pacientes: informação compartilhada como possível benefício terapêutico. Cad Saude Publica 2000; 16(3):815-822.

9. Souza TT, Silva WB, Onofre ASC, Quintans JSS, Onofre FBM, Quintans-Júnior LJ. Evaluation of adherence to treatment by patients seen in a psychosocial care center in northeastern Brazil. Braz J Pharm Sci 2011; 47(4):787-795.

10. Bond C. The need for pharmacy practice research. Int Pharm Pract 2006; 14:1-2.

11. Angonesi D, Rennó MUP. Dispensação farmacêutica: proposta de um modelo para a prática. Cien Saude Colet 2011; 16(9):3883-3891.

12. Marin N, organizadora. Assistência farmacêutica para gerentes municipais. Rio de Janeiro: OPAS, OMS; 2003.

13. Oenning D, Oliveira BV, Blatt CR. Conhecimento dos pacientes sobre os medicamentos prescritos após consulta médica e dispensação. Cien Saude Colet 2011; 16(7):3277-3283.

14. Brasil. Ministério da Saúde (MS). Portaria SVS/MS $n^{\circ}$ 344, de 12 de maio de 1998. Aprova o Regulamento Técnico sobre substâncias e medicamentos sujeitos a controle especial. Diário Oficial da União 1998; 19 maio.

15. Andrade MF, Andrade RCG, Santos V. Prescrição de psicotrópicos: avaliação das informações contidas em receitas e notificações. Rev. Bras. Cienc. Farm. 2004; 40(4):471-479.
16. Muijrers PEM, Knottnerus JA, Sijbrandij J, Janknegt R, Grol RPTM. Pharmacists in primary care. Pharm World Sci 2004; 26:256-262.

17. Gastelurrutia MA, Benrimoj SI, Castrillon CC, de Amezua MJ, Fernandez-Llimos F, Faus MJ. Facilitators for practice change in Spanish community pharmacy. Pharm World Sci 2009; 31:32-39.

18. Montgomery AT, Kälvemark-Sporrong S, Henning M, Tully MP, Kettis-Lindblad A. Implementation of a pharmaceutical care service: prescriptionists', pharmacists' and doctors' views. Pharm World Sci 2007; 29:593 602.

19. Vieira FS. Possibilidades de contribuição do farmacêutico para a promoção da saúde. Cien Saude Colet 2007; 12(1):213-220.

20. Brasil. Resolução do Conselho Nacional de Educação/ Câmara de Educação Superior no 2, de 19 de Fevereiro de 2002. Institui Diretrizes Curriculares Nacionais do Curso de Graduação em Farmácia. Diário Oficial da União 2002; 4 de mar.

21. Bell JS, Whitehead P, Aslani P, McLachlan AJ, Chen TF. Drug-related problems in the community setting: pharmacists' findings and recommendations for people with mental illnesses. Clin Drug Investig 2006; 26(7):415-425

22. Marques LA, Galduroz JC, Noto AR. Pharmaceutical care to patients treated with antidepressants. Rev Calid Asist 2012; 27(1):55-64.

23. Lizer MH, Parnapy Jawaid SA, Marsh W, Mogili L. The impact of a pharmacist assisted clinic upon medication adherence and quality of life in mental health patients. Pharmacy Practice 2011; 9(3):122-127.

24. Phokeo V, Sproule B, Raman-Wilms L. Community pharmacists' attitudes toward and professional interactions with users of psychiatric medication. Psychiatr Serv 2004; 55(12):1434-1436

25. Velligan DI, Weiden PJ, Sajatovic M, Scott J, Carpenter D, Ross R, Docherty JP. Strategies for addressing adherence problems in patients with serious and persistent mental illness: recommendations from the expert consensus guidelines. J Psychiatr Pract 2010; 16(5):306-324

26. Alencar TOS, Cavalcante EAB, Alencar, BR. Assistência farmacêutica e saúde mental no Sistema Único de Saúde. Rev Ciênc Farm Básica Apl 2012; 33(4):489-495

27. Brasil. Ministério da Saúde (MS). Conselho Nacional de Saúde. Relatório Final da VI Conferência Nacional de Saúde Mental - Intersetorial [Internet]. Brasília: MS; 2010.

Artigo apresentado em 24/09/2013

Aprovado em 06/01/2014

Versão final apresentada em 09/01/2014 